\title{
How Emotional Context Influences Facial Preferences and Impressions
}

\author{
Satomi Hara ${ }^{1,2}$, Kentaro Katahira ${ }^{3,4}$, Kazuo Okanoya ${ }^{2,3,4,5^{*}}$ \\ ${ }^{1}$ College of Arts and Sciences, The University of Tokyo, Tokyo, Japan \\ ${ }^{2}$ ERATO, Okanoya Emotional Information Project, Japan Science Technology Agency, Saitama, Japan \\ ${ }^{3}$ Center for Evolutionary Cognitive Sciences, The University of Tokyo, Tokyo, Japan \\ ${ }^{4}$ Emotional Information Joint Research Laboratory, RIKEN Brain Science Institute, Saitama, Japan \\ ${ }^{5}$ Graduate School of Arts and Sciences, The University of Tokyo, Tokyo, Japan \\ Email: "kazuookanoya@gmail.com
}

Received July $9^{\text {th }}, 2013$; revised August $12^{\text {th }}, 2013$; accepted September $16^{\text {th }}, 2013$

Copyright (c) 2013 Satomi Hara et al. This is an open access article distributed under the Creative Commons Attribution License, which permits unrestricted use, distribution, and reproduction in any medium, provided the original work is properly cited.

\begin{abstract}
Individuals change their evaluations of human faces depending on the valence of the stimuli presented with the faces. The present study investigated whether repeatedly presenting picture stimuli in pairs would evoke various emotions that would influence the subjects' preferences for and impressions of facial stimuli. The subjects' preferences for the facial stimuli did not differ consistently between before and after the facial stimuli were presented in tandem with emotional pictures. The direction of the change differed depending on the sex of the participant and the sex of face stimulus, even when the face was paired with a picture of the same valence. The emotional pictures appeared to have an effect on the emotions experienced in response to the face stimuli: the male participants, who were likely to feel pleasant emotions toward the pleasant pictures, were also likely to feel positive emotions toward the face stimuli paired with those pictures. Moreover, the female participants, who were likely to feel unpleasant emotions toward the unpleasant pictures, were also likely to feel afraid of the male faces paired with those pictures. These results suggest that the ability of an emotional stimulus to affect our preferences for and impressions of a face stimulus, as well as the degree of this effect, are highly sensitive to factors such as the sex of the participant and the sex of facial stimulus.
\end{abstract}

Keywords: Facial Preference; Facial Impression; Emotional Pictures; Evaluative Conditioning

\section{Introduction}

Preference is a critical factor that determines human behavior in various daily situations. Preference has a great influence on many aspects of our everyday decisions, such as what to eat, with whom to spend time, and what to buy. Thus, knowing how preferences are formed and manipulated is closely related to understanding human behavior and decision-making. To address this, many researchers have been studying a phenomenon called evaluative conditioning (EC). EC is a specific form of classical conditioning that refers to a change (either positive or negative) in one's evaluation of an initially neutral stimulus (a conditioned stimulus: CS) due to the valence of a stimulus presented in tandem with the CS (an unconditioned stimulus: US). Since EC was first observed by researchers such as Razran (1954) and Staats \& Staats (1957), a wide range of areas of psychology, including social psychology (e.g., Olson \& Fazio, 2001), learning psychology (e.g., Baeyens, Eelen, Crombez, \& Van den Bergh, 1992), and consumer psychology (e.g., Bierley, McSweeney, \& Vannieuwkerk, 1985), have been focused on this phenomenon.

Researchers have used stimuli of several domains, such as visual, auditory, and gustatory, to show that diverse types of

\footnotetext{
"Corresponding author.
}

stimuli can function as CS or US (see De Houwer, Thomas, \& Baeyens, 2001 for a review). Many of these studies have adopted pictures of human faces as CS. Baeyens et al. (1992) showed that faces initially rated as neutral became preferred after they were presented in pairs with highly preferred faces, and less preferred when presented with faces with low preference scores. Additionally, Hermans, Vansteenwegen, Crombez, Baeyens, \& Eelen (2002) used electric shocks and adjectives that described either positive or negative personalities as US and found that the preference scores for the faces paired with the negative adjectives or with the electric shocks decreased, whereas the preference scores for the faces paired with positive adjectives increased.

However, the emotions and impressions that people experienced in their everyday lives are much more complicated than simple terms such as "like", "dislike", "good" or "bad" or the disgust and avoidance produced by temporary punishments. In various situations, we become happy, angry, and sad and feel much more diverse emotions. Therefore, to profoundly understand how preferences are formed and manipulated in daily and social contexts, one must consider the influence of these types of complex and various emotions on the formation of preference. Nevertheless, no research has yet shown that such emotional stimuli can change individual preferences for CS. 
Therefore, in this study, we investigated whether US that induce complex and various types of emotions could change subjects' preferences for paired CS. We used the International Affective Picture System (IAPS) developed by Lang, Bradley \& Cuthbert (2008) as US. The IAPS is a database of pictures depicting a wide range of human experiences, which are scored using three criteria: valence, arousal, and dominance. Although this database was originally standardized using Western samples, many studies of Japanese samples (e.g., Katahira, Fujimura, Okanoya, \& Okada, 2011; Fujimura, Katahira, \& Okanoya, 2013) have confirmed that the images evoke similar emotions in individuals from both cultures. For the CS, we used pictures of human faces, which are socially important. We investigated whether the subjects' preferences for these faces would change according to the valence of IAPS pictures that were repeatedly paired with the faces. Although it has been confirmed that pleasant IAPS pictures can serve as appetitive stimuli and unpleasant IAPS pictures can be aversive stimuli in an instrumental conditioning paradigm (Katahira, Fujimura, Okanoya, \& Okada, 2011), it is not yet known whether these pictures can function as US in the EC paradigm.

There were four between-subject conditions that involved pairing the emotional pictures and the face stimuli: three experimental conditions and one control condition. The experimental conditions involved pairing a face stimulus with either a pleasant emotional picture (pleasant condition), an unpleasant emotional picture (unpleasant condition), or with a pleasant picture in half of the repetitions and with an unpleasant picture in the other half (pleasant-unpleasant condition). The control condition involved pairing a face stimulus with a picture with no particular emotional valence (neutral condition). Thus, we investigated how the subjects' preferences for the faces in each condition differed before and after the face stimulus and the emotional picture were presented in tandem. We hypothesized that the emotional pictures would have the same effect as the other stimuli in previous studies: the faces in the pleasant condition were expected to be preferred, those in the unpleasant condition to be less preferred, and those in the neutral condition to remain neutral. The pleasant-unpleasant condition is a novel condition created for the current study, and we expected it to affect preferences in three possible ways. First, faces in this condition might be greatly preferred due to the enhancement of the effect of the pleasant pictures by the unpleasant pictures. Second, the opposite pattern might occur: faces in this condition might be much less preferred due to the enhancement of the effect of the unpleasant pictures by the pleasant pictures. Third, the preference might not change due to a neutralizing effect between the unpleasant and pleasant pictures. We asked the subjects to what degree they felt a particular type of emotion, such as sadness or happiness, in response to the face stimuli after the conditioning. The responses to this question were used to assess the relationship among the emotions induced by the pictures, the changes in the preference rating, and the emotions evoked by the face stimuli.

\section{Methods}

\section{Participants}

A total of 53 Japanese university students (26 male and 27 female) participated in the study. The average age was 19.2 years $(\mathrm{SD}=.76$, range $=18-20)$. All the experimental procedures were approved by the Ethics Committee of the University of Tokyo.

\section{Stimuli}

The face stimuli ( 8 males and 8 females) were selected from a database that consisted of 49 male and 51 female faces, according to the data generated by a pilot study. The database was developed by the JST ERATO Okanoya Emotional Information Project (Fujimura and Okanoya, unpublished). In the pilot study, 11 participants aged 16 - 25 years (6 male and 5 female, average age 21.4 years) were shown each of the 100 face pictures in the database and rated how much they liked each face one a score of 1 to 9 (1: do not like it at all; 5: neutral; 9: like it very much). Among the pictures of comparatively young faces, we chose those that constantly received ratings close to 5 . The emotional pictures (30 pleasant, 30 unpleasant, and 20 neutral) were selected from the IAPS database using the valence ratings and arousal data in Lang et al. (2008).

\section{Procedures}

The experimental program was created with Inquisit ver. 3.0.6.0 by Millisecond Software and was presented on an LCD monitor. The participants were required to perform the necessary tasks by pressing a keyboard.

The experiment consisted of 4 parts: a pre-conditioning preference rating phase, a conditioning phase, a post-conditioning preference rating phase, and an emotion rating phase.

In the pre-conditioning preference rating phase, we measured the baseline preference of each participant for each face stimulus using a method similar to those of Field (2006) and Johnsrude, Owen, Zhao \& White (1999). We presented 2 of the 8 male or female face stimuli in turn, and we asked the participants to state which stimulus was preferred. Each of the possible pairs was presented twice: one on the left and the other on the right the first time, and the former on the right and the latter on the left the second time. Thus, each participant performed 112 trials in total. The preferred stimulus in each trial received 1 point, and the other received 0 ; thus, each face stimulus received a total of 0 to 14 points. The order between and within the male and female stimuli was randomized for each participant. This binary rating method was considered superior because when the participants were asked to rate the same stimuli again in the post-conditioning phase, they would be less likely to be influenced by memories of the ratings they had given to the stimuli in the pre-conditioning phase.

During the conditioning phase, we repeatedly presented each face stimulus paired with an emotional picture. The combinations used to create pairs of these two stimuli consisted of 3 experimental conditions and 1 control condition. The experimental conditions involved pairing a face stimulus with either a pleasant emotional picture (pleasant condition), an unpleasant emotional picture (unpleasant condition), or a pleasant picture in half of the repetitions and an unpleasant picture in the other half (pleasant-unpleasant condition). The control involved pairing a face stimulus with a picture with no particular emotional valence (neutral condition). Eight male and female face stimuli were divided into the 4 conditions so that each condition contains one male face and one female face. The assignment of face stimuli was counterbalanced between the participants.

The flow of one trial is shown in Figure 1. First, a face stimulus appeared on either the right or left side of the screen. 


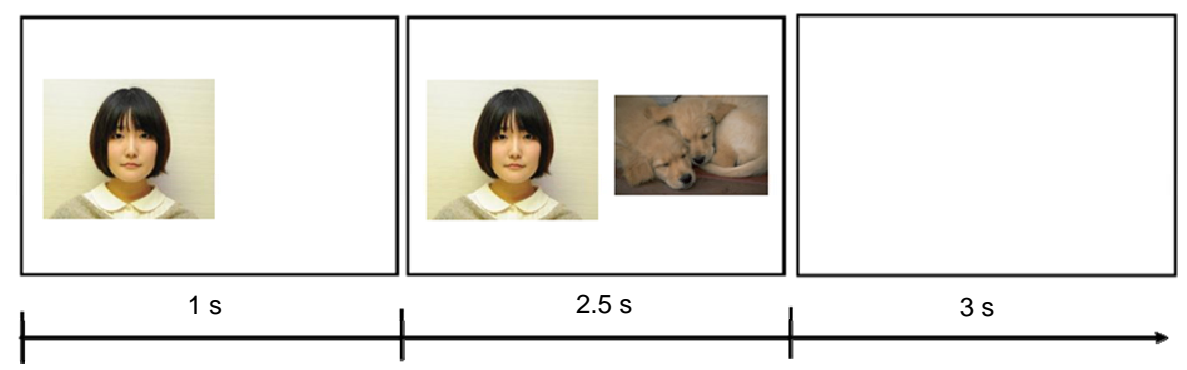

Figure 1.

The flow of the screen in the conditioning phase. The sample face stimulus and emotional picture were taken from other source materials to avoid showing materials of the databases used in the present study.

One second later, an emotional picture appeared on the opposite side of the screen. After another 2.5 seconds, these two stimuli disappeared simultaneously, and the screen remained empty for 3 seconds. During this interval, the participant rated the subjective level of pleasantness or unpleasantness of the emotional picture that had been presented on a scale of 1 to 9 (1: very unpleasant; 5: neutral; 9: very pleasant). After the interval, the next trial began.

Each face stimulus was presented in tandem with an emotional picture 10 times, the number that was expected to produce the most effective conditioning according to Baeyens et al. (1992). The face stimuli in the pleasant, unpleasant, and neutral conditions were paired with 10 different pleasant, unpleasant, and neutral pictures, respectively. The face stimuli in the pleasant-unpleasant condition were paired with 5 pleasant pictures and 5 unpleasant pictures. Each of the 30 pleasant, 30 unpleasant, and 20 neutral pictures was presented twice, once with a male stimulus and once with a female stimulus. Thus, each participant performed 160 trials in total. All the trials were divided into 5 sessions, for a total of 32 trials, and between the sessions, the participants could take a break of the desired length. Because the order in which the stimuli were presented and the timing of the rating were somewhat complicated, there was a practice session of 13 trials before the 5 experimental sessions. The practice session employed different face stimuli and emotional pictures from those used in the 5 experimental sessions.

In the post-conditioning preference rating phase, we measured the preference (after conditioning) for each face stimulus following the same method as in the pre-conditioning phase.

In the emotion rating phase, we investigated whether and to what degree the participants felt emotions while observing the face stimuli. The face stimuli were presented singly beneath one of 4 words: "sad", "scared", "happy", or "delighted", and the participants were asked to what degree they experienced the feeling of the depicted word while observing the face (1: not at all; 5: very much). Each of the 16 stimuli ( 8 male and 8 female) was presented with each of the 4 emotional words, for a total of 64 trials. The order in which the face stimuli and the words within each face were presented was randomized for each participant.

\section{Statistical Analyses}

$\mathrm{R}$ and anovakun ver. 4.3.1, an ANOVA function that runs on $\mathrm{R}$ software, was used for the statistical analyses. The significance level was set at $\alpha=.05$ for the ANOVA, and post-hoc comparisons were conducted using a modified Bonferroni method.

\section{Results}

\section{Ratings for the Emotional Pictures}

A 2 (sex of participant: male vs. female) $\times 3$ (type of emotional picture: pleasant, unpleasant, or neutral) ANOVA was used to analyze the ratings for the emotional pictures in the conditioning phase. The results revealed a significant main effect of the type of emotional picture $(F(2,102)=733.59, p$ $<.001)$ and a significant sex $\times$ type interaction $(F(2,102)=$ $15.49, p<.001)$. The post-hoc comparison of the sex $\times$ type interaction (Figure 2) revealed that both the male and female participants gave significantly higher ratings (more pleasant) to the neutral pictures than to the unpleasant ones (male: $F(1,51=$ $16.55, p<.001$; female: $F(1,51)=18.29, p<.001)$. Similarly, both sexes gave significantly higher ratings to the pleasant pictures than to the neutral ones (male: $F(1,51)=14.09, p<.001$, female: $F(1,51)=21.82, p<.001)$ or to the unpleasant ones (male: $F(1,51)=17.43, p<.001$, female: $F(1,51)=22.93, p$ $<.001)$. The female participants gave significantly higher ratings to the pleasant pictures and significantly lower ratings to the unpleasant ones compared with the male participants (pleasant: $F(1,51)=17.77, p<.001$; unpleasant: $F(1,51)=$ $10.11, p<.01)$. In addition, a one-sample t-test performed to compare the ratings given to the neutral pictures by the male and female participants revealed no significant difference from the most neutral rating, 5 (male: $t(25)=-.87, p=.39$; female: $t(26)=1.55, p=.13)$. These results suggest that all of the pleasant, unpleasant and neutral pictures had the expected valence for both the male and female participants. A comparison of the male and female participants, however, revealed that the females rated the pleasant pictures more positively and the unpleasant pictures more negatively than the males, suggesting that the emotional pictures may have produced a somewhat greater effect on the females than on the males.

\section{Preference Ratings for the Face Stimuli}

A 2 (sex of participant: male vs. female) $\times 2$ (sex of stimulus: male vs. female) $\times 4$ (condition: pleasant, unpleasant, pleasantunpleasant, or neutral) $\times 2$ (time: pre-conditioning vs. postconditioning) ANOVA was performed to analyze the preference ratings for the face stimuli. The results showed a significant sex of stimulus $\times$ condition $\times$ time interaction $(F(3,312)=$ $12.77, p<.05$ ), although none of the 8 conditions (sex of stimulus [2] $\times$ condition [4]) differed significantly between the pre-conditioning and the post-conditioning ratings, according to the post-hoc comparison. Figure 3 shows the average preference scores calculated for the male and female participants, 


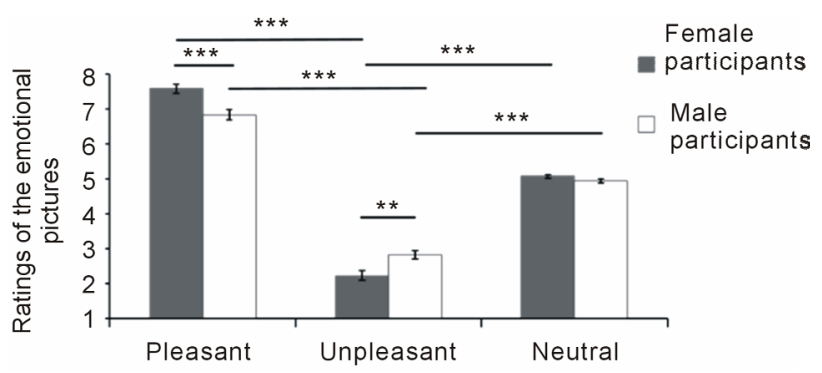

Figure 2.

The average valence rating of the emotional pictures. The error bars represent the s.e.m. ${ }^{* *} p<.01,{ }^{* * *} p<.001$. (a) Pleasant condition

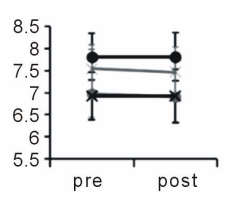

(c) Pleasant-unpleasant condition

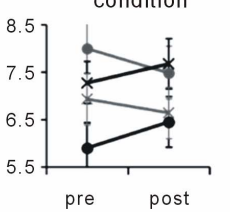

(b) Unpleasant condition

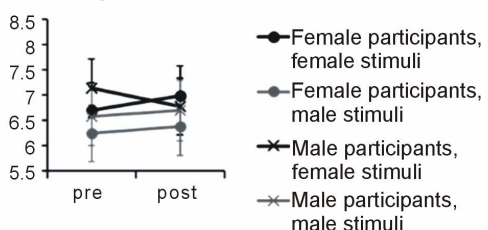

(d) Neutral condition

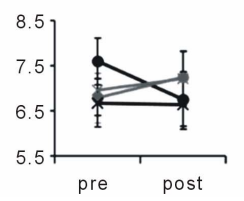

Figure 3.

The difference between the pre- and post-conditioning preference scores for the face stimuli. The error bars represent the s.e.m.

the male and female face stimuli, the conditions of pairing, and the pre-conditioning and post-conditioning ratings. These results indicate that the preference scores did not change in the same direction within each condition. Especially in the pleasant-unpleasant condition, the female faces received higher scores and the male face received lower scores in the postconditioning rating compared with the pre-conditioning rating (although these differences did not reach statistical significance).

In addition, to examine the possibility that the pre-conditioning score (minimum: 0, maximum: 14) affected the change in the preference score (post-conditioning score minus preconditioning score), we calculated the Pearson product-moment correlation coefficient between the pre-conditioning scores and the averages of the change in the preference scores; the result showed a strongly negative correlation $(n=15, r=-.92, p$ $<.001$; Figure 4). This result indicates that the face stimuli that received lower scores in the pre-conditioning rating tended to be rated more highly in the post-conditioning phase. The opposite was also true: the face stimuli that received higher scores in the pre-conditioning rating tended to receive lower scores in the post-conditioning rating.

\section{Emotional Ratings of the Face Stimuli}

A 2 (sex of participant: male vs. female) $\times 2$ (sex of stimulus: male vs. female) $\times 4$ (condition: pleasant, unpleasant, pleasant-unpleasant, or neutral) $\times 4$ (rated emotion: sad, scared, happy, or delighted) ANOVA was used to analyze the emo- tional ratings for the face stimuli. The results showed a significant main effect of the rated emotion $(F(3,312)=4.14, p<.01)$ and significant interactions between the sex of the participant $x$ emotion $(F(3,312)=2.65, p<.05)$, the sex of the stimulus $\times$ emotion $(F(3,312)=5.08, p<.01)$ and the sex of the participant $\times$ the sex of the stimulus $\times$ emotion $(F(3,312)=7.47, p$ $<.001)$. The post-hoc comparisons of the cubic interaction revealed different patterns between the male and female participants (Figure 5). The female participants evaluated the male and female stimuli differently (Figure 5(a)): for "sad" $(F(1,53)$ $=6.44, p<.05)$ and "scared" $(F(1,53)=11.76, p<.01)$, they gave higher ratings to the male stimuli than to the female stimuli; oppositely, for "happy" $(F(1,53)=15.02, p<.001)$ and "delighted" $(F(1,53)=10.21, p<.01)$, the female participants gave higher ratings to the females than to the males. The ratings of the male participants, in contrast, did not differ between the male and female stimuli (Figure 5(b)). This finding indicates that the female participants were more likely to experience positive emotions toward the female stimuli and negative emotions toward the male stimuli, whereas the male participants felt the same way toward both the male and female stimuli.

The lack of any significant effects of the paired picture condition (pleasant, unpleasant, pleasant-unpleasant, or neutral) indicates that there was no significant difference in the emotional ratings among the conditions, although the emotional rating was conducted after the conditioning process. However,

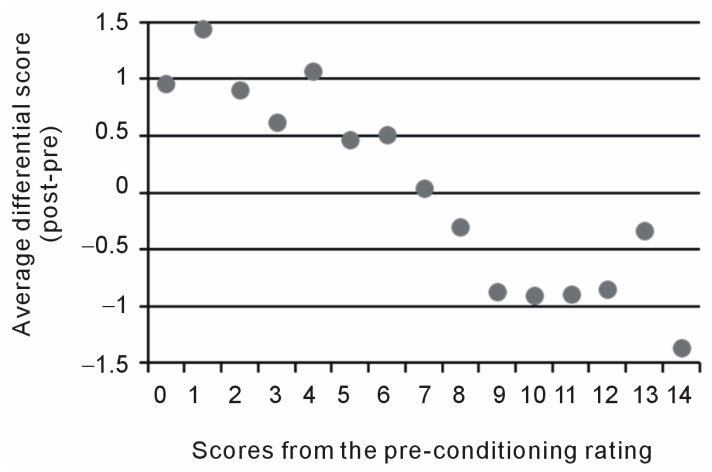

Figure 4.

The correlation between the pre-conditioning scores and the average change in the preference scores (from post-conditioning score to pre-conditioning score).

(a) Female participants

(b) Male participants

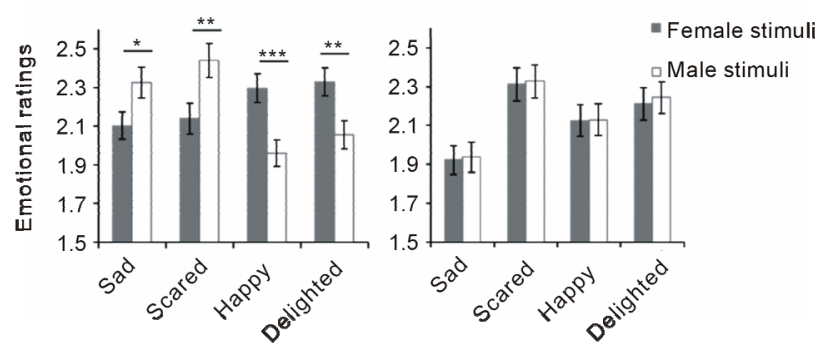

Figure 5.

The difference between males and females in the emotions experienced toward the face stimuli. The female participants felt strongly positive emotions in response to female stimuli and negative emotions in response to male stimuli. In contrast, the male participants felt the same way toward both the male and female stimuli. The error bars represent the s.e.m. ${ }^{*} p<.05,{ }^{* *} p<.01,{ }^{* * *} p<.001$. 
the degree to which a participant experiences an emotional picture as pleasant or unpleasant varies between individuals. There was a noteworthy correlation between the pleasant-unpleasant ratings for the emotional pictures and the emotional ratings for the face stimuli. The correlation was calculated separately for the sex of the participant and the stimulus and between the personal averages of the emotional ratings for the 4 kinds of emotion and those of the ratings for the emotional pictures that were paired with each face stimulus in the conditioning phase. As a result, the male participants produced partially consistent correlations irrespective of the sex of the face stimulus (Figures 6(a) and (b)): with regard to the pleasant condition face stimuli, the emotional ratings for "happy" and "delighted" showed positive correlations with the ratings for the pleasant pictures paired with those face stimuli (male stimulus, "happy"; $n=26, r=.40, p<.05$ : male stimulus, "delighted"; $n$ $=26, r=.26, p=.20$ : female stimulus, "happy"; $n=26, r=.42$, $p<.05$ : female stimulus, "delighted"; $n=26, r=.43, p<.05$ : Figure 6(a)). With regard to the pleasant-unpleasant condition face stimuli, the emotional ratings for "happy" and "delighted" were also positively correlated with the ratings for the pleasant pictures paired with those face stimuli (male stimulus, "happy"; $n=26, r=.31, p=.13$ : male stimulus, "delighted"; $n=26, r$ $=.52, p<.01$ : female stimulus, "happy"; $n=26, r=.52, p$ $<.01$ : female stimulus, "delighted"; $n=26, r=.48, p<.05$ : Figure 6(b)). That is, in both conditions in which the face stimuli were paired with pleasant pictures at least 5 times, the more that participants experienced pleasant emotions in response to the pleasant pictures, the more they experienced positive emotions in response to the face stimuli paired with those pictures. In contrast, the female participants' responses to the male stimuli of the unpleasant and pleasant-unpleasant conditions exhibited negative correlations between the emotional ratings for "scared" and the ratings for the unpleasant pictures (a smaller value indicates a more unpleasant response) that were paired with them (unpleasant condition; $n=27, r=-.38$, $p<.05$ : pleasant-unpleasant condition; $n=27, r=-.38, p<.05$ : Figure 6(c)). In both conditions in which the male stimuli were paired with unpleasant pictures at least 5 times, the more that the female participants experienced unpleasant emotions in response to the unpleasant pictures, the more they felt "scared" of the male stimuli that were paired with those pictures.

\section{Discussion}

The purpose of the current study was to consider the influence of emotional pictures on the preference for face stimuli presented in tandem with the emotional pictures and the emotions experienced toward such stimuli. Based on the hypothesis that the emotional pictures would produce the same effects as those of stimuli used in previous studies, the face stimuli that were paired with pleasant pictures (pleasant condition) were predicted to be preferred, and those paired with unpleasant pictures (unpleasant condition) were predicted to be less preferred. One of the purposes of this study was to investigate the effects of face stimuli paired with both pleasant and unpleasant pictures.

However, the preference scores for the face stimuli in each condition did not differ significantly before and after the conditioning process. Therefore, we would primarily like to discuss
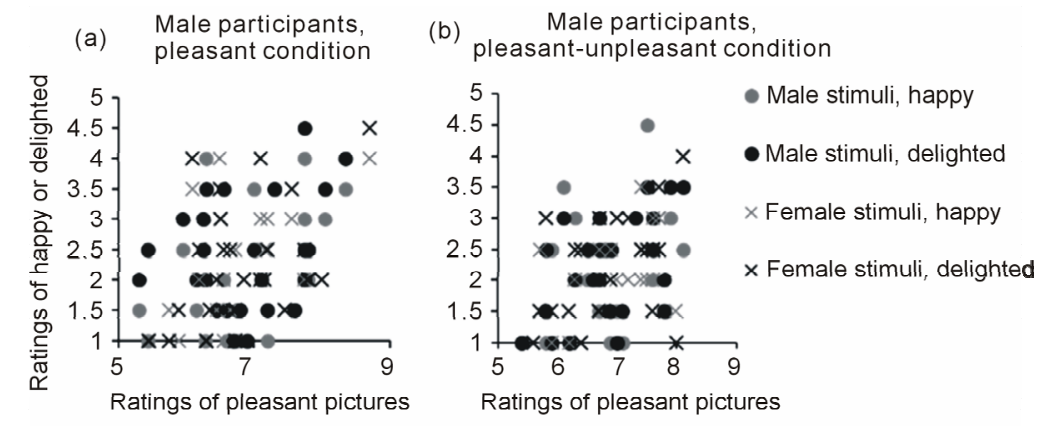

(c) Female participants, unpleasant

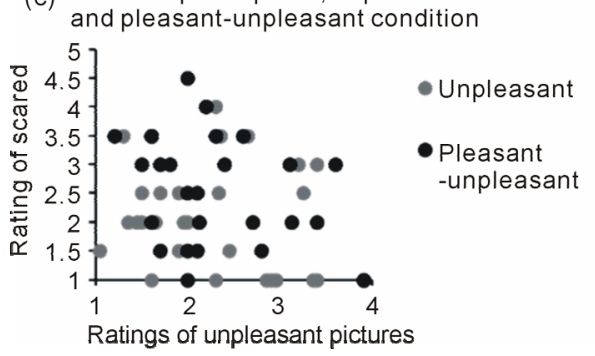

Figure 6.

The correlation between subjective emotional reactions to emotional pictures and to face stimuli after the conditioning process. Panels $\mathrm{a}$ and $\mathrm{b}$ show the responses of the male participants to both the male and female face stimuli; panel c shows the response of the female participants to the male stimuli alone. This figure indicates that the more that the male participants experienced pleasant emotions in response to the pleasant pictures, the more they experienced positive emotions in response to the face stimuli paired with those pictures. The more that the female participants experienced unpleasant emotions in response to the unpleasant pictures, the more they felt "scared" of the male stimuli paired with those pictures. 
the reasons for the results differing from our expectations. One possible reason is the considerable variance in the pre-conditioning scores. In previous studies that adopted human face stimuli as CS (Baeyens et al., 1992; Hermans et al., 2002), the participants were asked to evaluate many more face stimuli before the conditioning process than in this study; the purpose was to enable the researchers to choose as the CS the face with the most neutral score for each participant. This method was likely chosen because preferences for human faces are more susceptible to individual differences than are other stimuli. Previous studies thus employed a truly neutral face stimulus for each participant; therefore, there was little difference between the preference scores before conditioning. In contrast, we selected in advance face stimuli with stably neutral ratings by performing a pilot study and used them for all of the participants in the main experiment. Therefore, the preference scores for the face stimuli prior to conditioning varied considerably, depending on the participant and the stimulus. In addition, even the average scores within each of the 4 conditions were not equal: the average score in the pleasant condition was slightly higher than 7 (the middle score), and the average score in the unpleasant condition was slightly lower than 7 .

The strongly negative correlation between the pre-conditioning scores and the variation in the preference scores provide evidence that the above factor contributed to the difference between the results and the expected patterns. There are several possible causes of the correlation. A bias may have been introduced by the phenomenon that when the minimum score is limited, those who receive a low score in the first measurement will be more likely to receive a higher score in the second measurement, and vice versa. It is also possible that initially high or low evaluations of faces are difficult to manipulate or are likely to change in a definite direction (first high, then negative and first low, then positive), irrespective of the valence of the paired US (pleasant or unpleasant). Regardless of the cause, it is likely that the variance in the pre-conditioning ratings and the differences among the averages in each condition influenced the variations, as this correlation indicates, and thus made the direction of the preference change in each condition unclear.

Nonetheless, it is quite suspicious that we observed such a simple effect as expected although the influence stated above was excluded, considering that there were no constant tendencies at all within the conditions such that the preference scores of the pleasant condition face stimuli increased as those of the unpleasant condition face stimuli decreased. This finding suggests that the influence of emotional stimuli on preferences for faces is not necessarily identical to the influence of simple positive or negative stimuli, which were used in the previous studies. Emotional pictures may produce different effects, depending on the sex of the participant and the stimulus.

The results of the emotional ratings, on the other hand, suggest that the emotional pictures influenced the emotions experienced in response to the face stimuli. The male participants who were likely to feel pleasant emotions toward the pleasant pictures were also likely to feel positive emotions, such as "happy" and "delighted", toward the face stimuli in the pleasant and pleasant-unpleasant conditions, in which the face stimuli were paired with pleasant pictures at least 5 times. The female participants who were likely to experience unpleasant emotions toward the unpleasant pictures were also likely to feel "scared" of the male face stimuli in the unpleasant and pleasant-un- pleasant conditions, in which the face stimuli were paired with unpleasant pictures at least 5 times. This difference between the sexes suggests that the emotions that are readily attached to faces by conditioning may vary between males and females: males may be susceptible to conditioning between pleasant emotions and face stimuli, whereas females may be susceptible to conditioning between unpleasant emotions and male faces in particular, which must be avoided for safety under certain circumstances.

Moreover, the female participants experienced positive emotions, such as "happy" and "delighted", more strongly in response to the female face stimuli than to the male face stimuli. The females also experienced negative emotions, such as "sad" and "scared", more strongly in response to the male stimuli than to the female stimuli. The male participants, however, exhibited almost identical responses to the male and female stimuli. This result can be interpreted in several possible ways: it may reflect how males and females initially feel in response to unknown face stimuli or how they feel after unknown face stimuli are presented in tandem with emotional pictures, regardless of their valence. However, both interpretations indicate that this difference between the sexes reflects differences between males and females in the conditioning effect of emotional pictures on faces.

In this study, the emotional pictures produced no significant changes in the preference for the face stimuli presented in pairs with themselves. The influence of the emotional pictures on this preference may not have been as powerful and simple as the effects of the stimuli used in the previous studies, such as the liked and the disliked stimuli and the electric shocks, which changed the preference for CS in a constant direction irrespective of the sex of the participant or the stimulus. Concerning the emotions felt in response to the face stimuli, the emotional pictures did have an effect, the strength of which may have depended on the sex of the participant and the stimulus.

Therefore, to investigate how emotion-evoking stimuli affect our preferences and impressions of other stimuli, we must perform detailed analyses that consider such conditions. Additionally, larger samples than that of the present study are necessary to examine the difference between the sexes. In future research, more sophisticated experimental procedures and detailed analyses will be needed to clarify the relationship between stimuli that evoke complicated emotions and our preferences and emotions in response to other people and things.

\section{Acknowledgements}

We thank Maiko Fujimori for providing us with the database of facial stimuli. This work was supported in part by funding from the Japan Science and Technology Agency, Exploratory Research for Advanced Technology, Okanoya Emotional Information Project, and Grants-in-Aid for Scientific Research, no. 24700238 .

\section{REFERENCES}

Baeyens, F., Eelen, P., Crombez, G., \& Van den Bergh, O. (1992). Human evaluative conditioning: Acquisition trials, presentation schedule, evaluative style and contingency awareness. Behaviour Research and Therapy, 30, 133-142. http://dx.doi.org/10.1016/0005-7967(92)90136-5

Bierley, C., McSweeney, F. K., \& Vannieuwkerk, R. (1985). Classical conditioning of preferences for stimuli. Journal of Consumer Re- 
search, 12, 316-323. http://dx.doi.org/10.1086/208518

De Houwer, J., Thomas, S., \& Baeyens, F. (2001). Associative learning of likes and dislikes: A review of 25 years of research on human evaluative conditioning. Psychological Bulletin, 127, 853-869. http://dx.doi.org/10.1037/0033-2909.127.6.853

Field, A. P., (2006). I don't like it because it eats sprouts: Conditioning preferences in children. Behaviour Research and Therapy, 44, 439455. http://dx.doi.org/10.1016/j.brat.2005.03.006

Fujimura, T., Katahira, K., \& Okanoya, K. (2013). Contextual modulation of physiological and psychological responses triggered by emotional stimuli. Frontiers in Psychology, 4, 212.

http://dx.doi.org/10.3389/fpsyg.2013.00212

Hermans, D., Vansteenwegen, D., Crombez, G., Baeyens, F., \& Eelen, P. (2002). Expectancy-learning and evaluative learning in human classical conditioning: Affective priming as an indirect and unobtrusive measure of conditioned stimulus valence. Behaviour Research and Therapy, 40, 217-234.

http://dx.doi.org/10.1016/S0005-7967(01)00006-7

Johnsrude, I. S., Owen, A. M., Zhao W. V., \& White, N. M. (1999).
Conditioned preference in humans: A novel experimental approach. Learning and Motivation, 30, 250-264.

http://dx.doi.org/10.1006/lmot.1999.1031

Katahira, K., Fujimura, T., Okanoya, K., \& Okada, M. (2011). Decision-making based on emotional images. Frontiers in Psychology, 2, 311. http://dx.doi.org/10.3389/fpsyg.2011.00311

Lang, P. J., Bradley, M. M., \& Cuthbert, B. N. (2008). International affective picture system (IAPS): Affective ratings of pictures and instruction manual. Technical Report A-8, Gainesville, FL: University of Florida.

Olson, M. A., \& Fazio, R. H. (2001). Implicit attitude formation through classical conditioning. Psychological Science, 12, 413-417. http://dx.doi.org/10.1111/1467-9280.00376

Razran, G. (1954). The conditioned evocation of attitudes (cognitive conditioning?). Journal of Experimental Psychology, 48, 278-282. http://dx.doi.org/10.1037/h0058778

Staats, A. W., \& Staats, C. K. (1957). Meaning established by classical conditioning. Journal of Experimental Psychology, 54, 74-80. http://dx.doi.org/10.1037/h0047716 